\title{
A Carbon Nanotube Field Effect Transistor-Based Circuit of Electrophysiology Retina in Neural Applications
}

\author{
Ala'aDdin Al Shidaifat', Jae Keun Song ${ }^{2 *}$, Yeji Han ${ }^{3}$, Yong Su Park ${ }^{4}$, Hanjung Song ${ }^{5}$ \\ 1,3,5Inje University, Department of Nanoscience and Engineering \\ ${ }^{2 *}$ Department of physical therapy \\ 197 Inje-ro, Gimahe, Republic of Korea \\ alaaddinsh@hotmail.com; hjsong@inje.ac.kr \\ ${ }^{4}$ Chung Cheong University, Department of Electronic Engineering \\ 38 Wolgok-gil, Cheongju, Republic of Korea \\ yspark@ok.ac.kr
}

\section{Extended Abstract}

The main goal of understanding neural systems is to develop prosthetic devices which can replace by lesioned neural devices in a near future. The first time-independent neuron model was proposed by McCulloch and Pitts in 1943. McCulloch and Pitts tried to understand how the brain could produce highly complex patterns by using many basic cells that are connected together. These basic brain cells are called neurons. Hodgkin and Huxley have contributed into a lot of researches; one of the most important research for them was in 1952, in that work they uncovered the electrical properties of neurons and implemented it in electronic circuits [1]. In the last three decades, the development of biological system has grown and many researchers attempt to emulate neural networks that are characterized by parallel processing and low power consumption. Mead and Mahowald demonstrated a new imaging paradigm that mimicked the human retina with silicon integrated circuits in 1988. The silicon retina has been produced by them is providing the first glimpse of the great potential of CMOS integrated circuits technology for imaging [2]. For many years, engineers and scientists have been using the metaloxide semiconductor field-effect transistors (MOSFETs) as basic elements to design integrated circuit (IC). They have used the MOSFET in their circuit for various reasons like: cheaper to fabricate and consume lesser power [3]. The scaling process in IC designs is achieved by reducing the size of a transistor. The scaling process which is used in designing the chip is characterized by properties, such as high density, low power, and high speed. The continuous scaling of the circuit design causing several problems like: power dissipation, process variation, short channel effect and leakage current [3]. The CNT was discovered as a solution for many problems of MOSFET with similar performance achievement. In 1991, the theory of nanotubes was observed by Ijima [4]. In this study, we propose a new integrated circuit of an I\&F (integrated and fire) model for electronic retina. The proposed circuit designed using carbon nanotube (CNT), the I\&F neuron model that consist of a photodiode for the light input, a capacitor for summing of input current signals, an amplifier with a feedback capacitor which makes neuron pulse and a photo sensor. The SPICE (simulation program with integrated circuit emphasis) results of the circuit in a condition of $0.9 \mathrm{~V}$ power supply demonstrate that the neuron outputs pulses with a small amplitude is around 70 $\mathrm{mV}$.

\section{Acknowledgment}

This research was supported by the Basic Science Research Program through the National Research Foundation of Korea (NRF) funded by the Ministry of Education, Science and Technology (NRF-2015R1D1A1A01057495).

\section{References}

[1] A. L. Hodgkin and A. F. Huxley, "The components of membrane conductance in the giant axon of Loligo," J. Physiol, vol. 116, no. 4, pp. 473-496, 1952.

[2] M. Mahowald and R. Douglas, "A silicon neuron," Nature, vol. 354, pp. 515-518, 1991.

[3] J. Appenzeller, J. Knoch, R. Martel, V. Derycke, S. J. Wind, and P. Avouris, "Carbon nanotube electronics," IEEE Trans. on Nanotechnol., vol. 1, no. 4, pp. 184-189, 2002. 
[4] S. Iijima and T. Ichihashi, "Single-shell carbon nanotubes of $1 \mathrm{~nm}$ diameter," Nature, vol. 363, pp. 603-605, 1991. 